\title{
Classification of line-transitive point-imprimitive linear spaces with line size at most 12
}

\author{
Cheryl E. Praeger \\ School of Mathematics and Statistics, \\ The University of Western Australia, Crawley, WA 6009, Australia \\ email: praeger@maths.uwa.edu.au \\ Shenglin Zhou \\ Department of Mathematics, Shantou University, \\ Shantou, Guangdong 515063, P. R. China \\ email:slzhou@stu.edu.cn
}

October 30, 2018

\begin{abstract}
In this paper we complete a classification of finite linear spaces $\mathcal{S}$ with line size at most 12 admitting a line-transitive point-imprimitive subgroup of automorphisms. The examples are the Desarguesian projective planes of orders 4,7,9 and 11, two designs on 91 points with line size 6 , and 467 designs on 729 points with line size 8 .
\end{abstract}

Keywords: linear spaces, line-transitive, point-imprimitive.

AMS subject classification: 05B05, 05B25, 20B25

\section{Introduction}

A finite linear space $\mathcal{S}=(\mathcal{P}, \mathcal{L})$ consists of a finite set $\mathcal{P}$ whose elements are called points, and a set $\mathcal{L}$ of subsets of $\mathcal{P}$ whose elements are called lines, such that each pair of points is contained in exactly one line and each line contains at least two points. It is said to be trivial if there is only one line, or if all lines have only two points, and otherwise it is called non-trivial. The automorphism group $\operatorname{Aut}(\mathcal{S})$ of $\mathcal{S}$ consists of all permutations of $\mathcal{P}$ that leave $\mathcal{L}$ invariant, and a subgroup $G$ of $\operatorname{Aut}(\mathcal{S})$ is said to be line-transitive if it acts transitively on $\mathcal{L}$. For line-transitive linear spaces the size of lines is constant, say $k$, and for non-trivial ones $2<k<v$, where $v=|\mathcal{P}|$. It is possible for a line-transitive group $G$ to leave invariant a non-trivial partition of $\mathcal{P}$, and in this case we say that $G$ is point-imprimitive on $\mathcal{S}$; otherwise $G$ is called point-primitive. In 1989, A. Delandtsheer and J. Doyen proved 
that, for a given value of the line size, there are only finitely many linear spaces that admit a line-transitive, point-imprimitive group. In 1996, A.R. Camina and S. Mischke ([4]) classified all line-transitive, point-imprimitive linear spaces with line size $k$ at most 8 , and recently such linear spaces with $k / \operatorname{gcd}(k, v) \leq 8$ were classified in [3]. Here we continue this work, and extend these classifications for line sizes up to 12. Building on the work in [3, 4, and the results of exhaustive computer searches described in [1, 2, 9, 16], we obtain the following classification.

Theorem 1.1 Let $\mathcal{S}$ be a non-trivial finite linear space with $v$ points and line size $k \leq 12$. Assume that $\mathcal{S}$ has a group of automorphisms which is line-transitive and point-imprimitive. Then $\mathcal{S}$ is one of the following:

(a) A Desarguesian projective plane $\mathrm{PG}_{2}(q)$ with $q \in\{4,7,9,11\}$;

(b) One of two linear spaces with $v=91$ and $k=6$, namely the Mills design or the Colbourn-McCalla design;

(c) One of 467 linear spaces with $v=729$ and $k=8$ constructed by Nickel, Niemeyer, O'Keefe, Penttila and Praeger in [16], namely the $N^{2} O P^{2}$ designs.

In all cases both the full automorphism group $\operatorname{Aut}(\mathcal{S})$ and all line-transitive, pointimprimitive subgroups are known. The main result that is proved in this paper is Theorem 1.2. Together with the work mentioned above and some non-trivial computer searches discussed in Remark 1.3, Theorem 1.2 yields Theorem 1.1 .

Theorem 1.2 Let $\mathcal{S}=(\mathcal{P}, \mathcal{L})$ be a non-trivial finite linear space, admitting a linetransitive, point-imprimitive group $G$ of automorphisms. Let $v=|\mathcal{P}|$, and suppose that the line size $k$ satisfies $9 \leq k \leq 12$ and $\operatorname{gcd}(k, v)=1$. Then $G$ has a line regular subgroup $H$, and $k, v, H$ satisfy one of the lines of Table 1. Moreover, in Lines 1,3-5 of Table 1, $a$ point stabilizer in $H$ fixes a unique point.

\begin{tabular}{|c|ccc|}
\hline Line & $k$ & $v$ & $H$ \\
\hline 1 & 9 & 217 & $\left(Z_{7} \times Z_{31}\right): Z_{3}$ \\
2 & 10 & 91 & $Z_{7} \times Z_{13}$ \\
3 & 10 & 451 & $\left(Z_{11} \times Z_{41}\right): Z_{5}$ \\
4 & 11 & 1431 & $\left(Z_{3}^{3} \times Z_{53}\right): Z_{13}$ \\
5 & 12 & 133 & $Z_{7} \times Z_{19}$ \\
\hline
\end{tabular}

Table 1: Results for Theorem 1.2 
Remark 1.3 (a) Not all of the lines of Table 1 lead to examples of line-transitive linear spaces. However this fact cannot easily be demonstrated theoretically and requires extensive, and exhaustive, computer searches. We summarise the results of those searches here. First, in the case of Line 3, there are no examples. This fact was proved by Greg Cresp [9] in his undergraduate honours research project. Next the spaces in Line 2 are projective planes, and the fact that the only projective plane admitting a line-transitive cyclic group of order 91 is the Desarguesian plane $\mathrm{PG}_{2}(9)$ was proved in [2, Lemma 4.5]. The main aim of [2] was to complete a classification initiated in [18, Theorem 1.6].

Similar but more delicate theory and extensive computation were required to prove that for Line 5, the only projective plane admitting a line-transitive cyclic group of order 133 is $\mathrm{PG}_{2}(11)$, and in Lines 1 and 4 there are no examples. The theory and computational techniques involved in the latter searches are as yet unpublished, and will be reported in [1. The results of these computer searches, together with Theorem 1.2 and [4, Theorem 1], complete the proof of Theorem 1.1.

(b) Comparable group theoretic information to that given in Table 1 is available for groups $G$ acting line-transitively and point-imprimitively on the linear spaces with line size $k \leq 8$ mentioned in Theorem 1.1. This is summarised in Table 2, where $F_{n}$ denotes a Frobenius group of order $n$, and $P$ is the relatively free, 3-generator, exponent 3, nilpotency class 2 group of order $3^{6}$. More details about the action of $G$ for the designs in the last line are provided in [16, Section 2].

\begin{tabular}{|c|c|c|}
\hline $\mathcal{S}$ & $G$ & Reference \\
\hline $\mathrm{PG}_{2}(4)$ & $H \times K$, where $H=Z_{3}$ or $S_{3}$ and & [17, Theorem 1.1] \\
& $K=Z_{7}$ or $F_{21}$ & \\
$\mathrm{PG}_{2}(7)$ & $H \times K$, where $H=Z_{3}$ and & [8, p. 50] \\
& $K=Z_{19}$ or $F_{57}$ & {$[19]$} \\
Mills & $G=Z_{91} \cdot Z_{d}$, where $d \mid 3$ & {$[19]$} \\
Colbourn-McCalla & $G=Z_{91} \cdot Z_{d}$, where $d \mid 12$ & [17, Theorem 1.2] and \\
NNOPP & $G=\operatorname{Aut}(\mathcal{S})=H \cdot Z_{13}$, where & [16, Main Theorem 1.3] \\
\hline
\end{tabular}

Table 2: Group information for Remark 1.3

One motivation for the research project reported in this paper was to exploit and test the power of the current linear space theory, as presented in [3]. The objective of the paper [3] was to collate the available theory of finite line-transitive, point-imprimitive linear spaces, refine it and develop it further, and then organise it into a series of algorithms that could be run on a computer. The output of the algorithms would be a (hopefully manageable) set 
of feasible parameters and putative line-transitive, point-imprimitive groups for such linear spaces. These algorithms were implemented, some in C and others in the GAP language [14], and were applied in [3] to classify all examples for which $k / \operatorname{gcd}(k, v) \leq 8$. In particular this showed that there were no examples with $9 \leq k \leq 12$ and $\operatorname{gcd}(k, v)>1$.

To prove Theorem [1.2, we used the algorithms in [3] to produce a list of feasible parameters and group theoretic information for examples with $9 \leq k \leq 12$ and $\operatorname{gcd}(k, v)=1$ (see Section 3). We then made a detailed analysis of each of these possibilities in Section 4. Some additional theoretical lemmas used in Section 4 are presented in Section 2.

\section{Preliminary Results}

\subsection{Notation and hypotheses}

Let $G$ be a transitive group of permutations on a set $\mathcal{P}$. A partition $\mathfrak{C}$ of $\mathcal{P}$ is $G$-invariant if, for all parts $C \in \mathfrak{C}$ and all $g \in G$, the image $C^{g}$ of $C$ under $g$ is also a part of $\mathfrak{C}$. For each $G$-invariant partition $\mathfrak{C}$ of $\mathcal{P}, G$ induces a transitive permutation group $G^{\mathfrak{C}}$ on $\mathfrak{C}$ (called the top group), and the setwise stabiliser $G_{C}$ of $C \in \mathfrak{C}$ induces a transitive permutation group $G^{C}$ on $C$ (called the bottom group). Moreover, $G^{C}$ is independent of the choice of $C$ in $\mathfrak{C}$ up to permutation isomorphism. The kernel of $G$ on $\mathfrak{C}$ is the subgroup $G_{(\mathfrak{C})}$ of elements $g \in G$ with $C^{g}=C$ for each $C \in \mathfrak{C}$. Thus $G^{\mathfrak{C}} \cong G / G_{(\mathfrak{C})}$. We say that $\mathfrak{C}$ is $G$-normal if $G_{(\mathfrak{C})}$ is transitive on each of the classes of $\mathfrak{C}$.

For partitions $\mathfrak{C}, \mathfrak{C}^{\prime \prime}$ of $\mathcal{P}, \mathfrak{C}$ refines $\mathfrak{C}^{\prime \prime}$ if every class of $\mathfrak{C}$ is contained in a class of $\mathfrak{C}^{\prime \prime}$, and this refinement is strict if $\mathfrak{C} \neq \mathfrak{C}^{\prime}$. We also say that $\mathfrak{C}^{\prime}$ is coarser, strictly coarser than $\mathfrak{C}$, respectively. Let $\mathfrak{C}$ be a $G$-invariant partition of $\mathcal{P}$. Then $\mathfrak{C}$ is called minimal if the only strict $G$-invariant refinement is the discrete partition with all classes of size 1 ; and $\mathfrak{C}$ is maximal if the only $G$-invariant partition that is strictly coarser than $\mathfrak{C}$ is the all-in-one partition (with a single class). Equivalently, $\mathfrak{C}$ is minimal, maximal if and only if $G^{C}, G^{\mathfrak{C}}$ is primitive, respectively (where $C \in \mathfrak{C}$ ).

Throughout the paper we assume the following Hypotheses. Here we denote by $\operatorname{Soc}(G)$ the socle of a group $G$, that is, the product of the minimal normal subgroups of $G$.

Hypotheses. (a) Let $\mathcal{S}=(\mathcal{P}, \mathcal{L})$ be a finite linear space with $v$ points and $b$ lines, each of size $k$, and with $r$ lines through each point, where $2<k<v$.

(b) Assume that $\mathcal{S}$ admits a line-transitive, point-imprimitive subgroup $G$ of automorphisms which leaves invariant a non-trivial partition $\mathfrak{C}=\left\{C_{1}, \ldots, C_{d}\right\}$ of $\mathcal{P}$ with $d$ classes of size $c$ where $c>1$ and $d>1$, so that $v=c d$ and, by [11],

$$
c=\frac{\left(\begin{array}{l}
k \\
2
\end{array}\right)-x}{y} \quad \text { and } \quad d=\frac{\left(\begin{array}{l}
k \\
2
\end{array}\right)-y}{x}
$$

with $x, y$ (called the Delandtsheer-Doyen parameters) positive integers. Let $\alpha$ denote a point in $\mathcal{P}$, and let $C \in \mathfrak{C}$ be the class containing $\alpha$. 
(c) Let the $\mathfrak{C}$-intersection type be $\left(1^{d_{1}}, \cdots, k^{d_{k}}\right)$, that is, for each $i$, each line meets $d_{i}$ classes of $\mathfrak{C}$ in $i$ points, and $\sum_{i} i d_{i}=k$.

(d) If $\mathfrak{C}$ is $G$-normal, let $K:=G_{(\mathfrak{C})}, S:=\operatorname{Soc}(K), X:=C_{G}(K)$, and $Y:=C_{G}(S)$.

\subsection{Some lemmas}

First we state some results from Camina and Siemons [6] and Camina and Praeger [5].

Lemma 2.1 Assume that the Hypotheses hold.

(a) [6, Lemma 4] Each involution in $G$ fixes at least one point.

(b) [6, Lemma 5] Suppose that $G$ has a point-regular normal subgroup $M$. Then no element of $G$ induces the inversion map $x \mapsto x^{-1}$ on $M$.

(c) [5, Theorem 1] If $N \unlhd G$ then $N$ acts faithfully on each of its orbits in $\mathcal{P}$.

The next lemma extends [3, Lemma 8.2].

Lemma 2.2 Assume that the Hypotheses hold and that $\mathfrak{C}$ is $G$-normal and minimal.

(a) Then $\mathfrak{C}$ is the set of $S$-orbits in $\mathcal{P}$ and

(i) Either $Y \cap K=1$, or $S$ is elementary abelian and $Y \cap K=S$.

(ii) Either $X \cap K=1$, or $S$ is elementary abelian and $X \cap K=K=S$.

(b) Suppose in addition that $\mathfrak{C}$ is maximal, that $Y \cap K=S \neq Y$, and that one of the following conditions holds.

\begin{tabular}{|c|c|c|}
\hline Condition & $\operatorname{Soc}\left(G^{\mathfrak{C}}\right)$ & Extra Property \\
\hline 1 & abelian & $\operatorname{gcd}(c, d)=1$ \\
2 & non-abelian & $\begin{array}{c}\text { Schur multiplier of a minimal normal subgroup } \\
\text { of } G^{\mathfrak{C}} \text { has no section isomorphic to } S\end{array}$ \\
\hline
\end{tabular}

Then $G$ has a normal subgroup $M=T \times S$ where $T$ is a minimal normal subgroup of $G$ and $T^{\mathfrak{C}}$ is minimal normal in $G^{\mathfrak{C}}$. Moreover either

(i) $T$ is non-abelian and transitive on $\mathcal{P}$, or

(ii) the set $\mathfrak{C}^{\prime \prime}$ of $T$-orbits in $\mathcal{P}$ is a G-normal partition of $\mathcal{P}$ with $\left|\mathfrak{C}^{\prime}\right|=c$ such that for $C \in \mathfrak{C}$ and $C^{\prime} \in \mathfrak{C}^{\prime \prime},\left|C^{\prime}\right|=d$ and $\left|C \cap C^{\prime}\right|=1$. Moreover either $M$ is regular on $\mathcal{P}$ or $T$ is not semiregular on $\mathcal{P}$. 
Proof. Part (a) is proved in [3, Lemma 8.2]. For part (b), since $Y \cap K=S$, it follows from part (a)(i) that $S$ is abelian, and since $\mathfrak{C}$ is minimal the bottom group $G^{C}$ is primitive. By Lemma 2.1(c), $S^{C} \cong S$, and since $S^{C}$ is normal in $G^{C}$, it follows that $S^{C}$ is regular and is a minimal normal subgroup of $G^{C}$. Hence $S$ is a minimal normal subgroup of $G$. By assumption $Y \not \leq K$ and hence $Y^{\mathfrak{C}}$ is a non-trivial normal subgroup of $G^{\mathfrak{C}}$, and since $\mathfrak{C}$ is maximal, $G^{\mathfrak{C}}$ is primitive. Thus there exists a subgroup $M \triangleleft G$ such that $S<M \leq Y$ and $M^{\mathfrak{C}} \cong M / S$ is a minimal normal subgroup of $G^{\mathfrak{C}}$. Suppose first that condition 1 holds. Then $\operatorname{Soc}\left(G^{\mathfrak{C}}\right)=M^{\mathfrak{C}}, d=p^{a}$ for some prime $p$, and a Sylow $p$-subgroup $T$ of $M$ is $Z_{p}^{a}$ (since $\operatorname{gcd}(c, d)=1)$. Since $T \leq C_{G}(S), T$ is characteristic in $M$, and hence $T \triangleleft G$, and $M=T \times S$ is regular on $\mathcal{P}$. By [3, Lemma 8.2], the $T$-orbits form a $G$-normal partition of $\mathcal{P}$ with the properties of (b)(ii). Since $T^{\mathfrak{C}}$ is minimal normal in $G^{\mathfrak{C}}$, it follows that $T \cong T^{\mathfrak{C}}$ is minimal normal in $G$, so b(ii) holds.

Now suppose that condition 2 holds. Let $T=M^{\prime}$, the derived subgroup of $M$. Since $M^{\mathfrak{C}}=M / S$ is a minimal normal subgroup of $G^{\mathfrak{C}}$ and $\operatorname{Soc}\left(G^{\mathfrak{C}}\right)$ is non-abelian, $M / S$ has no non-trivial abelian quotient, and so $T^{\mathfrak{C}}=M^{\mathfrak{C}}$. Also, since $M \leq Y=C_{G}(S)$, we have $T \cap S \leq M^{\prime} \cap Z(M)$, that is, $T \cap S$ is contained in the Schur multiplier of $T^{\mathfrak{C}}$. Moreover, since $S$ is minimal normal in $G$ either $T \cap S=1$ or $T \cap S=S$. By condition 2, the second possibility cannot occur. So $T \cap S=1$, and $M=T \times S$ with $T$ minimal normal in $G$.

Since $T^{\mathfrak{C}}$ is transitive, each $T$-orbit $D$ in $\mathcal{P}$ meets each $\mathfrak{C}$-class in a constant number of points, say $c_{0}$, and for $C \in \mathfrak{C}, D \cap C$ is a block of imprimitivity for $G$, and hence also for $G^{C}$, of size $c_{0}$. Since $G^{C}$ is primitive, $c_{0}=1$ or $c$. Thus $T$-orbits in $\mathcal{P}$ have length $d$ or $v$ respectively. In the latter case $T$ is transitive, and $\mathrm{b}(\mathrm{i})$ holds. In the former case, by [3, Lemma 8.2], the set of $T$-orbits forms a $G$-normal partition of $\mathcal{P}$ with the properties of (b)(ii). Finally, either $M$ is regular on $\mathcal{P}$ or $T$ is not semiregular on $\mathcal{P}$.

Lemma 2.3 Suppose that the Hypotheses hold, and also that part (b)(ii) of Lemma 2.2 holds with $M$ acting regularly on $\mathcal{P}$. Then we may identify $\mathcal{P}$ with $M=\{(t, s) \mid t \in T, s \in S\}$ such that, for $\alpha=\left(1_{T}, 1_{S}\right), G \leq \hat{G}:=M \cdot(\operatorname{Aut}(T) \times \operatorname{Aut}(S))$ and $G_{\alpha} \leq \hat{G}_{\alpha}=\operatorname{Aut}(T) \times$ $\operatorname{Aut}(S)$ acting naturally on $M$. Moreover,

$$
\begin{aligned}
& \mathfrak{C}=\left\{C_{t} \mid t \in T\right\} \quad \text { where } \quad C_{t}=\{(t, s) \mid s \in S\} \quad \text { for } t \in T \\
& \mathfrak{C}^{\prime}=\left\{C_{s}^{\prime} \mid s \in S\right\} \quad \text { where } \quad C_{s}^{\prime}=\{(t, s) \mid t \in T\} \quad \text { for } s \in S
\end{aligned}
$$

$K=S .\left(G_{\alpha} \cap \operatorname{Aut}(S)\right)$ and the kernel $L$ of the $G$-action on $\mathfrak{C}^{\prime \prime}$ is $L=T .\left(G_{\alpha} \cap \operatorname{Aut}(T)\right)$.

Proof. Suppose that $M=T \times S$ is regular on $\mathcal{P}$, and set $X:=M$.Aut $(M)$. Then we can identify $M$ with $\mathcal{P}$ such that $G \leq X \leq \operatorname{Sym}(\mathcal{P})$, and for $\alpha=\left(1_{T}, 1_{S}\right), G=M G_{\alpha}$ with $G_{\alpha} \leq X_{\alpha}=\operatorname{Aut}(M)$ acting naturally on $M$. Moreover, since $T, S \triangleleft G$ we have $G_{\alpha} \leq N:=$ $N_{\text {Aut }(M)}(T) \cap N_{\operatorname{Aut}(M)}(S)$. Now each $\sigma \in N$ induces $\sigma^{T} \in \operatorname{Aut}(T)$ and $\sigma^{S} \in \operatorname{Aut}(S)$ and we have a natural homomorphism $\varphi: N \rightarrow \operatorname{Aut}(T) \times \operatorname{Aut}(S)$ defined by $\varphi(\sigma)=\left(\sigma^{T}, \sigma^{S}\right)$. In fact $\varphi$ is an isomorphism so we may identify $N$ with $\operatorname{Aut}(T) \times \operatorname{Aut}(S)$. Setting $\hat{G}=M . N$ we have $\hat{G}_{\alpha}=N$. Finally, since $\mathfrak{C}, \mathfrak{C}^{\prime}$ are the sets of $S$-orbits and $T$-orbits in $\mathcal{P}$, respectively, (2) and (3) hold, and we have $K=S .\left(G_{\alpha} \cap \operatorname{Aut}(S)\right)$ and $L=T .\left(G_{\alpha} \cap \operatorname{Aut}(T)\right)$. 
The last lemma of the section addresses a special case of Lemma 2.2 (b)(ii) that arises frequently in our search.

Lemma 2.4 Suppose that the Hypotheses hold, and also that part (b)(ii) of Lemma 2.2 holds with $M$ regular on $\mathcal{P}$ and $c$ and d distinct odd primes. Then $T:=\langle t\rangle \cong Z_{d}, S:=\langle s\rangle \cong$ $Z_{c}, \operatorname{Aut}(T)=\langle g\rangle \cong Z_{d-1}, \operatorname{Aut}(S)=\langle h\rangle \cong Z_{c-1}, G_{\alpha} \leq\langle g\rangle \times\langle h\rangle$. Let $g^{\prime}=g^{\frac{d-1}{2}}, h^{\prime}=h^{\frac{c-1}{2}}$. Then we may re-label the classes of $\mathfrak{C}$ and $\mathfrak{C}^{\prime}$ so that

$$
\begin{aligned}
& \mathfrak{C}=\left\{C_{i} \mid 0 \leq i \leq d-1\right\} \quad \text { where } \quad C_{i}=\left\{\left(t^{i}, s^{j}\right) \mid 0 \leq j \leq c-1\right\} \\
& \mathfrak{C}^{\prime}=\left\{C_{j}^{\prime} \mid 0 \leq j \leq c-1\right\} \quad \text { where } C_{j}^{\prime}=\left\{\left(t^{i}, s^{j}\right) \mid 0 \leq i \leq d-1\right\}
\end{aligned}
$$

and in particular $C_{0}=S$ and $C_{0}^{\prime}=T$. Moreover,

(a) (i) $\operatorname{Fix}_{\mathcal{P}}\left(g^{\prime}\right)=C_{M}\left(g^{\prime}\right)=S \in \mathfrak{C}, \operatorname{Fix}_{\mathcal{P}}\left(h^{\prime}\right)=C_{M}\left(h^{\prime}\right)=T \in \mathfrak{C}^{\prime \prime}$.

(ii) $g^{\prime} h^{\prime} \notin G_{\alpha}$, and $G_{\alpha}$ contains at most one of $g^{\prime}, h^{\prime}$. In particular a Sylow 2-subgroup of $G$ is cyclic.

(b) If $|G|$ is even then one of (i)-(iv) below holds.

(i) $x \leq 8<y$ and $g^{\prime} \in G_{\alpha}$, $\operatorname{Fix}_{\mathcal{P}}\left(g^{\prime}\right)=S \in \mathfrak{C}$,

(ii) $y \leq 8<x$ and $h^{\prime} \in G_{\alpha}, \operatorname{Fix}_{\mathcal{P}}\left(h^{\prime}\right)=T \in \mathfrak{C}^{\prime}$,

(iii) $x \geq 9$ and $y \geq 9$,

(iv) $x \leq 8$ and $y \leq 8$, and $\mathcal{S}$ is $\mathrm{PG}_{2}(4), \mathrm{PG}_{2}(9), \mathrm{PG}_{2}(11)$, or the Colbourn-McCalla design.

Proof. The first assertions, as well as (4) and (5), follow from Lemma 2.3. Moreover, as $\hat{G}$ acts naturally on $\mathcal{P}=M$, part (a)(i) holds. Since $g^{\prime} h^{\prime}$ acts as the inversion map on $M$, Lemma 2.1 (b) implies that $g^{\prime} h^{\prime} \notin G_{\alpha}$. Thus $G_{\alpha}$ contains at most one of $g^{\prime}$ and $h^{\prime}$. Then, since $\left|G: G_{\alpha}\right|=c d$ is odd and $G_{\alpha} \leq\langle g\rangle \times\langle h\rangle$, it follows that $G_{\alpha}$ contains a Sylow 2-subgroup of $G$ and a Sylow 2-subgroup is cyclic.

Now suppose that $|G|$ is even. By the previous paragraph, it follows that $G_{\alpha}$ contains exactly one involution $w$, and that $w$ is $g^{\prime}$ or $h^{\prime}$. Let $F=\operatorname{Fix}_{\mathcal{P}}(w)$. By (a)(i), $F$ is $S$ or $T$ respectively. If both $x>8$ and $y>8$ then (b)(iii) holds, so assume that at least one of $x, y$ is at most 8. If $w=h^{\prime}$ then $K$ contains $S .\left\langle h^{\prime}\right\rangle$ and so $K$ is not semiregular on $\mathcal{P}$. Then, by [18, Theorem 1.6] and [2, Theorem 1.6], either $x>8$ (in which case we must have $y \leq 8$ and (b)(ii) holds), or $x \leq 8$ and $\mathcal{S}$ is as in (b)(iv) and for these linear spaces $y \leq 8$ also. Similarly if $w=g^{\prime}$ then $L$ contains $T \cdot\left\langle g^{\prime}\right\rangle$ and so $L$ is not semiregular on $\mathcal{P}$. Again, either $y>8$ (in which case we must have $x \leq 8$ and (b)(i) holds), or $x, y \leq 8$ and $\mathcal{S}$ is as in (b)(iv). 


\section{Running the algorithms}

Assume that the Hypotheses hold with $9 \leq k \leq 12$ and $\operatorname{gcd}(k, v)=1$. As explained in [3, Section 3], to find all pairs $(\mathcal{S}, G)$ we may assume that either $G$ is quasiprimitive on $\mathcal{P}$ (that is to say, all non-trivial normal subgroups of $G$ are transitive on $\mathcal{P}$ ), or $\mathfrak{C}$ is $G$-normal. The search procedures in [3] return parameter and group information for all possibilities $(\mathcal{S}, G)$ with $k / \operatorname{gcd}(k, v)$ at most a given maximum value $k_{\max }^{(r)}$. We applied Algorithms 1, 6, 7 and 8 from [3] assuming that $9 \leq k \leq k_{\max }^{(r)}=12$ and $\operatorname{gcd}(k, v)=1$. We are grateful to Dr. Maska Law for performing these computer tests for us. The only possibilities for the parameters $v=c \cdot d, k, x, y$ and the intersection type were those given in Table 3 . Other parameter information returned by these algorithms is not shown as it is not needed for our further analysis in Section 4. The algorithms also returned the group theoretic information given in Table 4 about the top group $G^{\mathfrak{C}}$ and the bottom group $G^{C}$. In particular the computer tests proved the following:

(1) In all Lines of Table 3 the partition $\mathfrak{C}$ is $G$-normal (that is, there were no possiblities with $G$ quasiprimitive).

(2) In all Lines of Table 3, $\mathfrak{C}$ is maximal and minimal, so both $G^{\mathfrak{C}}$ and $G^{C}$ are primitive.

\section{Detailed Analysis}

Throughout this Section, we assume that the Hypotheses hold and that $9 \leq k \leq 12$. Thus one of the Lines in Tables 3 and 4 holds. We give a detailed group theoretic analysis for each of these Lines, thus proving Theorem 1.2. In the following, "Line $i$ " will denote the $i$-th line of Tables 3 and 4 . First we deal completely with Line 15 and obtain some preliminary information for the other LINES.

Lemma 4.1 (a) If $S=Z_{c}$ and $d \nmid(c-1)$, then $Y \cap K=S \neq Y$ and $Y^{\mathfrak{C}}$ is transitive.

(b) Line 15 does not give any examples, and moreover one of (i)-(iii) holds.

(i) One of LINES $7-11$ holds, $G$ has a point and line regular cyclic subgroup $Z_{7} \times Z_{13}$ and $\mathcal{S}=\mathrm{PG}_{2}(9)$.

(ii) One of LINES 26 - 31 or $32-35$ holds and $G$ has a point-regular normal cyclic subgroup $Z_{7} \times Z_{19}$ or $Z_{5} \times Z_{53}$, respectively. In LINES $26-31$, $\mathcal{S}$ is a projective plane of order 11 .

(iii) One of Lines $1-6,12-14,16-25,36-38$ holds, and $K=S \cong \operatorname{Soc}\left(G^{C}\right)$. 
Proof. (a) Recall (from Section 3) that $\mathfrak{C}$ is maximal and minimal. Suppose that $S=Z_{c}$ and $d \nmid(c-1)$. Since $G^{C}$ is primitive on $C$ with socle $Z_{c}, c$ must be a prime. Hence $G / Y \leq \operatorname{Aut}(S)=Z_{c-1}$. So $d \nmid|G / Y|$, and therefore $Y^{\mathfrak{C}}$ is a non-trivial normal subgroup of the primitive group $G^{\mathfrak{C}}$, so $Y^{\mathfrak{C}}$ is transitive. In particular $Y \neq Y \cap K$. Also by Lemma 2.1(c), $K \cong K^{C} \leq \mathrm{AGL}(1, c)$, so $Y \cap K=C_{K}(S)=S$.

(b) We deal with Line 15 in (b)(ii) below.

(b)(i) LinEs 7-11: Here $\mathcal{S}$ is a projective plane and in all these Lines the parameter $x$ is 3 or 6 . By Table 4, $S=\operatorname{Soc}(K) \cong Z_{c}$, and $d \nmid(c-1)$. Thus by part (a), $Y \cap K=S$ and $Y^{\mathfrak{C}}$ is transitive. Hence $Y$ contains an element $g$ of order a power of $d$ such that $\langle g\rangle^{\mathfrak{C}}$ is transitive. Then $g^{d} \in Y \cap K=S$ and is a $d$-element, so $g^{d}=1$. Thus $\langle g, S\rangle \cong Z_{91}$ is regular on points and lines of $\mathcal{S}$. By [2, Lemma 4.5], $\mathcal{S}$ is the Desarguesian projective plane $\mathrm{PG}_{2}(9)$.

(b)(ii) Lines 15 and 26-35: In each of these Lines, $S=Z_{c}$ is as in Table 4, and $d \nmid(c-1)$. So by part (a), $Y \cap K=S \neq Y$ and $Y^{\mathfrak{C}}$ is transitive. Hence $Y^{\mathfrak{C}}$ contains $\operatorname{Soc}\left(G^{\mathfrak{C}}\right)$ since in all cases $G^{\mathfrak{C}}$ has a unique minimal normal subgroup. In all cases the conditions of Lemma 2.2(b) hold and so we obtain $T \leq Y, T \triangleleft G$ and $T \cong \operatorname{Soc}\left(G^{\mathfrak{c}}\right)$. Again, in all cases $c \nmid|T|$ so $T$ is intransitive with $c$ orbits of length $d$, and the $T$-orbits form another $G$-normal partition $\mathfrak{C}^{\prime}$ with $c$ classes of size $d$. In particular for Line 27, the parameters for $\mathfrak{C}^{\prime}$ should satisfy Line 30 or 31 , and so $T \cong Z_{7}$. Similarly, for Lines $32-34$, the parameters for $\mathfrak{C}^{\prime \prime}$ should satisfy LINE 35 , and so $T \cong Z_{5}$. Thus for all of the LinES under consideration, $T \cong Z_{d}$ and $M=T \times S$ is a point-regular normal cyclic subgroup of $G$. In LinEs 15 and 26-31, $b / v=1$ so $\mathcal{S}$ is a projective plane. However, for Line 15, there is no corresponding Line with parameters $(d, c)=(3,37)$, so Line 15 is ruled out. (Note that Line 15 corresponds to a projective plane of order 10, and it was shown in [15] that there is no such projective plane.)

(b) (iii) In all of these remaining LiNES, $x \leq 8$, and so by [18, Theorem 1.6], $K=S \cong$ $\operatorname{Soc}\left(G^{C}\right)$.

Note that, by Lemma 4.1, Theorem 1.2 is proved if one of Lines $7-11,15$, or $26-31$ holds. Therefore from now on we will assume that one of the other LINES holds, namely LiNEs 1-6, $12-14,16-25$, or $32-38$. We next deal with LiNEs $20-23$, which required different arguments from the other LinEs.

Lemma 4.2 Lines $20-23$ give no examples.

Proof. Suppose that one of Lines $20-23$ holds and let $c=c_{0}^{2}, d=d_{0}^{2}$, where $c_{0}=7, d_{0}=3$ in the case of Lines 20-22, and $c_{0}=3, d_{0}=7$ in Line 23. By Lemma 4.1(b)(iii) and Table 3, $K=S=\operatorname{Soc}\left(G^{C}\right) \cong Z_{c_{0}}^{2}$ and so $G / Y \leq \mathrm{GL}\left(2, c_{0}\right)$. Since $S^{C} \cong S$ is self-centralising in $G^{C}$ it follows that $S^{C}=(Y \cap K)^{C} \cong Y \cap K$, and hence $Y \cap K=S$. We claim that $Y^{\mathfrak{C}} \neq 1$. Suppose to the contrary that $Y^{\mathfrak{C}}=1$. Then $Y=Y \cap K=S$. Thus $Y \leq K$ and hence $\left|G^{\mathfrak{C}}\right|$ divides $|G / Y|$, which in turn divides $\left|\mathrm{GL}\left(2, c_{0}\right)\right|$. In particular $d$ divides $\left|\mathrm{GL}\left(2, c_{0}\right)\right|$, and so $d=9$ and $d$ divides the order of $G / Y \leq \mathrm{GL}(2,7)$. Since 27 does not divide $|\mathrm{GL}(2,7)|$, and since $Y=S=Z_{7}^{2}$, it follows that a Sylow 3-subgroup $P$ of $G$ is isomorphic to a Sylow 3-subgroup $Z_{3} \times Z_{3}$ of $\mathrm{GL}(2,7)$. Thus $P$ has a subgroup $P_{0} \cong Z_{3}$ such that $P_{0} Y / Y \cong P_{0}$ is contained 
in the centre of $\mathrm{GL}(2,7)$. This implies that $P_{0} Y / Y \triangleleft G / Y$. Hence $P_{0} S=P_{0} Y \triangleleft G$ and so $P_{0}^{\mathfrak{C}}=\left(P_{0} S\right)^{\mathfrak{C}} \triangleleft G^{\mathfrak{C}}$. This is a contradiction since $\left|P_{0}^{\mathfrak{C}}\right|=3$ and all nontrivial normal subgroups of $G^{\mathfrak{C}}$ are transitive on $\mathfrak{C}$. Hence $Y^{\mathfrak{C}} \neq 1$ and the claim is proved. Now $Y \cap K=S$, so we have $S<M \leq Y$ with $M \triangleleft G$ and $M / S \cong M^{\mathfrak{C}} \cong \operatorname{Soc}\left(G^{\mathfrak{C}}\right)$.

CASE 1. $M^{\mathfrak{C}}=\operatorname{Soc}\left(G^{\mathfrak{C}}\right)=Z_{d_{0}}^{2}$. In this case $M$ is abelian with a unique Sylow $c_{0^{-}}$ subgroup $T=Z_{c_{0}}^{2}$ and $M=T \times S$. Moreover $M$ is regular on $\mathcal{P}, T$ is normal in $G$ and semiregular on $\mathcal{P}$, and the set of $T$-orbits forms a second $G$-normal partition $\mathfrak{C}^{\prime \prime}$ of $\mathcal{P}$, with $c$ parts of size $d$, for which one of Lines 20-23 holds. Thus, for the remainder of our consideration of this case, we may assume that $S=Z_{7}^{2}, T=Z_{3}^{2}$, and we may identify $\mathcal{P}$ with $M$ so that $M$ acts by right multiplication and, for $\alpha=1_{M}$, we have $G=M . G_{\alpha}$ with $G_{\alpha} \leq \operatorname{Aut}(M)=\mathrm{GL}(2,3) \times \mathrm{GL}(2,7)$ acting by conjugation.

Since 4 divides $b$, a Sylow 2-subgroup $Q$ of $G$ has order at least 4 , and since $v$ is odd, $Q$ fixes a point. Replacing $Q$ by a conjugate if necessary, we may assume that $Q$ fixes $\alpha$. Let $C \in \mathfrak{C}, C^{\prime} \in \mathfrak{C}^{\prime \prime}$ such that $C \cap C^{\prime}=\{\alpha\}$. Then $Q$ fixes $C, C^{\prime}$ setwise and we note that $\left|C^{\prime} \backslash\{\alpha\}\right|=8$. Since $4 \mid b$, all $Q$-orbits on $\mathcal{L}$ have length a multiple of 4 . In particular $Q$ fixes no lines and hence $\alpha$ is the unique fixed point of $Q$. Moreover, since for any point $\beta \neq \alpha, Q_{\alpha \beta}$ fixes the line $\lambda(\alpha, \beta)$ setwise, it follows that all $Q$-orbits in $\mathcal{P} \backslash\{\alpha\}$ have length a multiple of 4 . Since all entries in the $\mathfrak{C}$-intersection type are at most 4 , no element of $S$ of order 7 fixes a line, so $S$ is semiregular on $\mathcal{L}$. Similarly, since the $\mathfrak{C}^{\prime}$-intersection type is $\left(1^{9}, 2\right)$, no element of $T$ of order 3 fixes a line, and so $T$ is semiregular on lines. Thus $M$ is semiregular on $\mathcal{L}$ with 4 orbits of length $v$. It follows that $M Q$ is transitive on $\mathcal{L}$, so without loss of generality we may assume that $G=M Q$. Further, since each element of order 2 in $Q$ fixes some line, we have $|Q| \geq 8$.

If $Q \cap K \neq 1$, then $K$ is not semiregular, and Lemma 2.4(b)(iv) applies giving a contradiction. Thus $Q \cap K=1$ and so $Q \cong Q^{\mathfrak{C}} \leq \mathrm{GL}(2,3)$. Each Sylow 2-subgroup of $\mathrm{GL}(2,3)$ is isomorphic to $\Gamma \mathrm{L}(1,9)=Z_{8} \cdot 2$ and contains the central involution $z_{0}$ of $\mathrm{GL}(2,3)$. Then, since $|Q| \geq 8$, it follows that $Q$ contains an involution $z$ such that $z^{\mathfrak{C}}=z_{0}$, and therefore $\langle M, z\rangle$ is normal in $G$. If in addition $z^{\mathfrak{C}^{\prime \prime}} \neq 1$, then $z^{\mathfrak{C}^{\prime \prime}}$ is the central involution of $\operatorname{GL}(2,7)$, and so $z$ inverts each element of $M$, contradicting Lemma 2.1(a). Hence $z^{\mathfrak{C}^{\prime \prime}}=1$, and $G_{\left(\mathfrak{C}^{\prime}\right)}$ contains $\langle T, z\rangle$ which is not semiregular on $\mathcal{P}$. Again Lemma 2.4(b)(iv) applies and we have a contradiction.

CASE 2. $M^{\mathfrak{C}}$ is non-abelian.

CASE 2a. Lines 20-22 with $M^{\mathfrak{C}}=L_{2}(8)$ or $A_{9}$, or Line 23 with $M^{\mathfrak{C}}=L_{3}(2)^{2}$.

The Schur multiplier of $L_{2}(8), A_{9}$ or $L_{3}(2)^{2}$ is $1, Z_{2}$, or $Z_{2}^{2}$ respectively, so the conditions of Lemma 2.2(b) hold. Hence $G$ has a normal subgroup $T \times S$ where $T \cong M^{\mathfrak{c}}$, and either $T$ is transitive on $\mathcal{P}$ or the set of $T$-orbits forms a $G$-normal partition $\mathfrak{C}^{\prime}$ with $c$ parts of size $d$. In the latter case, parameters for the $G$-normal partition $\mathfrak{C}^{\prime}$ should satisfy Line 23 if one of Lines 20-22 holds for $\mathfrak{C}$, or one of the Lines 20-22 if Line 23 holds for $\mathfrak{C}$. In particular, by Table 3, $T$ should be abelian, which is a contradiction. Hence $T$ is transitive on $\mathcal{P}$, and in particular $c|| T \mid$. This implies that $c=9$ and $T=L_{3}(2)^{2}$. Then $|T|=2^{6} v$ and hence the stabiliser $T_{\alpha}$ in $T$ of a point $\alpha$ is a Sylow 2-subgroup of $T$. Thus $T_{\alpha}=\left(Z_{2}^{3}\right)^{2}$ and $N_{T}\left(T_{\alpha}\right)=\left(Z_{2}^{3} \cdot Z_{7}\right)^{2}$. By [13, Theorem 4.2A], the set $F$ of fixed points of $T_{\alpha}$ in $\mathcal{P}$ has size 
$\left|N_{T}\left(T_{\alpha}\right): T_{\alpha}\right|=49=\left|C_{\text {Sym }(\mathcal{P})}(T)\right|$ and $C_{\text {Sym }(\mathcal{P})}(T)$ acts regularly on $F$. However $S=Z_{3}^{2}$ centralises $T$ and cannot be a subgroup of a group of order 49 . So this case cannot arise.

CASE 2b. Line 23 with $M^{\mathfrak{C}}=A_{7}^{2}$.

Here $\mathfrak{C}=\mathfrak{C}_{1} \times \mathfrak{C}_{2}$, where $\mathfrak{C}=\left\{C_{i j} \mid 1 \leq i \leq 7,1 \leq j \leq 7\right\}$, and $A_{7}^{2} \leq G^{\mathfrak{C}} \leq S_{7} 2 S_{2}$ in product action. $\operatorname{Soc}\left(G^{\mathfrak{C}}\right)$ acts on $\mathfrak{C}$ as follows. Let $\left(h_{1}, h_{2}\right) \in A_{7}^{2} \leq G^{\mathfrak{C}}$, and $C_{i j} \in \mathfrak{C}$. Then $C_{i j}^{\left(h_{1}, h_{2}\right)}=C_{i^{h_{1}, j^{h_{2}}}}$. Let $P$ be a Sylow 5 -subgroup of $G$. Since $\operatorname{gcd}(5, b)=1, P$ is a Sylow 5 subgroup of $G_{\lambda}$ for some $\lambda \in \mathcal{L}$. Note that if $P$ fixes $C_{i j} \in \mathfrak{C}$ setwise, then since $|\operatorname{AGL}(2,3)|$ is not divisible by $5, P$ must fix $C_{i j}$ pointwise. Hence $\operatorname{Fix}_{\mathcal{P}}(P)$ is a union of $\mathfrak{C}_{\text {-classes. Without }}$ loss of generality, assume that $P^{\mathfrak{C}}=\langle(12345)\rangle \times\langle(12345)\rangle$, so $\operatorname{Fix}_{\mathfrak{C}}(P)=\left\{C_{i j} \mid i, j=6\right.$ or 7$\}$. Then $\left|\operatorname{Fix}_{\mathfrak{C}}(P)\right|=4$. Since the intersection type is $\left(1^{9}, 2\right)$ and since $P \leq G_{\lambda}, P$ fixes setwise the unique class, say $C$, with $|C \cap \lambda|=2$, and $P$ fixes setwise the 9 classes $C^{\prime}$ such that $\left|C^{\prime} \cap \lambda\right|=1$. Since $9 \equiv 4(\bmod 5), P$ must fix setwise at least 4 of these 9 classes and hence $P$ fixes at least $1+4=5$ classes setwise. This contradiction completes the proof.

We now show for all the remaining cases, namely LINES 1-6, 12-14, 16-19, 24-25 and $32-38$, that $G$ has a normal abelian point-regular subgroup.

Lemma 4.3 The group $G$ has a point-regular, normal abelian subgroup $M$, and either

(a) LiNe 6 holds, and $M=Z_{17} \times Z_{17}$ or $Z_{17^{2}}$, or

(b) $M=T \times S$, where

$$
S=\left\{\begin{array}{ll}
Z_{3}^{3} & \text { if LINE } 25 \text { holds } \\
Z_{c} & \text { for all other Lines }
\end{array} \quad \text { and } \quad T= \begin{cases}Z_{3}^{3} & \text { if LINE } 24 \text { holds } \\
Z_{d} & \text { for all other Lines }\end{cases}\right.
$$

Proof. For Lines 32-35 this is proved in Lemma 4.1 (b). Suppose therefore that one of the other Lines holds. Then, by Lemma 4.1 (b)(iii), $K=S=\operatorname{Soc}\left(G^{C}\right)$, and by Table 3, $S$ is $Z_{3}^{3}$ for Line 25, and $Z_{c}$ with $c$ prime for all the other Lines. For Line 25, $G / Y \leq \operatorname{Aut}(S)=\operatorname{GL}(3,3)$, and so $d=53$ does not divide $|G / Y|$. Hence in this case $Y \not K K$, and so $Y^{\mathfrak{C}} \neq 1$, which implies that $Y^{\mathfrak{C}}$ is transitive. For all the other LINES we also have $Y^{\mathfrak{C}}$ transitive, by Lemma 4.1 (a).

For LiNE 6 , since $K=S=Z_{17}$ and $Y^{\mathfrak{C}}$ is transitive, there exists a normal subgroup $M$ of $G$ such that $S<M \subseteq Y$ with $M / S=Z_{17}$. So $M=Z_{17} \times Z_{17}$ or $Z_{17^{2}}$, $M$ is pointregular, and (a) holds. In all the other LINES, $\operatorname{gcd}(c, d)=1$, and hence the conditions of Lemma 2.2(b) hold and we obtain a normal subgroup $M=T \times S$ of $G$, where $T$ is a minimal normal subgroup of $G$ and $T^{\mathfrak{C}} \cong T$ is minimal normal in $G^{\mathfrak{C}}$. In all cases $c \nmid|T|$, so $T$ is intransitive on $\mathcal{P}$ with $c$ orbits of length $d$. The $T$-orbits form a $G$-normal partition $\mathfrak{C}^{\prime \prime}$ with $c$ classes of size $d$, and the parameters for $\mathfrak{C}^{\prime}$ appear in one of the LINES in Table 3, according to the table below.

\begin{tabular}{|c|c|c|c|c|c|c|c|c|c|}
\hline LiNE for $\mathfrak{C}^{\prime}$ & $1-2$ & 3 & 4 & 5 & $12-13$ & 14 & $16-17$ & $18-19$ & 24 \\
LiNE for $\mathfrak{C}^{\prime \prime}$ & 3 & $1-2$ & 5 & 4 & 14 & $12-13$ & $18-19$ & $16-17$ & 25 \\
\hline
\end{tabular}


It follows from Table 3 that $T$ must be $Z_{d}$ for all Lines apart from Line 24 , where $T=Z_{3}^{3}$. In all LiNEs therefore $M=T \times S$ is point-regular, abelian, and normal.

The next lemma completes our analysis, and thereby completes the proof of Theorem 1.2 .

Lemma 4.4 (a) If one of LINES 4-5,12-14,24-25 holds then one of the Lines 1,3, 4 of Table 1 holds, respectively.

(b) Lines $1-3,6,16-19,32-38$ give no examples.

Proof. By Lemma 4.3, $G$ has an abelian point-regular normal subgroup $M$. Moreover in all Lines apart from Line $6, M=T \times S$ with $|T|=d$ and $|S|=c$, while for Line 6 either $M$ has this form with $c=d=17$, or $M \cong Z_{17^{2}}$. Note that $|G / M|$ is divisible by $b / v$, where $b / v$ is as in Table 3. We will identify $\mathcal{P}$ with $M$ so that $M$ acts by right multiplication, and taking $\alpha=1_{M}, G=M G_{\alpha}$ with $G_{\alpha} \leq \operatorname{Aut}(M)$ acting naturally.

First we deal with Line 6 in the case where $M=Z_{17^{2}}$. It is convenient here to take $M=$ $\mathcal{P}$ as the additive group of integers modulo 289 , so that $G_{\alpha} \leq \operatorname{Aut}(M)=\left\{\sigma_{i} \mid \operatorname{gcd}(17, i)=\right.$ $1\} \cong Z_{17} \cdot Z_{16}$, where $\sigma_{i}: a \longmapsto a i(a \in M)$. Since $b / v=4$ divides $\left|G_{\alpha}\right|, G_{\alpha}$ contains the involution $\sigma_{-1}$, and this contradicts Lemma 2.1 (b).

Hence $M=T \times S$ in all cases. Let $\mathfrak{C}$ be the set of $S$-orbits and $\mathfrak{C}^{\prime}$ the set of $T$-orbits, so that $\mathfrak{C}, \mathfrak{C}^{\prime}$ are $G$-invariant partitions of $\mathcal{P}$ with $|\mathfrak{C}|=d,\left|\mathfrak{C}^{\prime}\right|=c$. Let $K=G_{(\mathfrak{C})} \geq S$, and $L=G_{\left(\mathfrak{C}^{\prime}\right)} \geq T$.

Suppose first that one of the Lines 1-6, 12-14, 16-19, or 36-38 holds. Then, by Lemma 4.1, $K=S \cong Z_{c}$ and $L=T \cong Z_{d}$. Also by Lemma 2.4, we may set $T=\langle t\rangle \cong Z_{d}, S=\langle s\rangle \cong Z_{c}$, $\mathcal{P}=M, \alpha=(1,1), \operatorname{Aut}(T)=\langle g\rangle \cong Z_{d-1}, \operatorname{Aut}(S)=\langle h\rangle \cong Z_{c-1}$ and $G_{\alpha} \leq\langle g\rangle \times\langle h\rangle$. Since $K=S$ and $L=T$ we have $G_{\alpha} \cap\langle g\rangle=G_{\alpha} \cap\langle h\rangle=1$. Thus $G_{\alpha}$ is cyclic of order dividing $\operatorname{gcd}(c-1, d-1)$, and $b / v$ divides $|G / M|=\left|G_{\alpha}\right|$. In all of these LinEs, both $x \leq 8$ and $y \leq 8$, and it follows from Lemma 2.4 (b) that $|G|$ is odd, and in particular $b / v$ is odd. The only possibilities are LINES 4-5 with $G_{\alpha} \cong Z_{3}$, and LinEs 12-14 with $G_{\alpha} \cong Z_{5}$. In both cases $G$ is line-regular, and so Line 1 or 3 of Table 1 holds, respectively.

Now suppose that one of the Lines 24-25 holds. By Lemma 4.1, $K=S$ and $L=T$, and without loss of generality we may take $T=Z_{3}^{3}$, and $S=Z_{53}$. By Lemma 2.3, $G_{\alpha} \leq$ $\operatorname{Aut}(T) \times \operatorname{Aut}(S)=\mathrm{GL}(3,3) \times Z_{52}$ with $G_{\alpha} \cap \operatorname{Aut}(T)=G_{\alpha} \cap \operatorname{Aut}(S)=1$. In particular $G_{\alpha}$ is cyclic of order dividing 52 and divisible by $b / v=13$. Thus $G$ has a line-regular subgroup $H=M . Z_{13}$ with $H_{\alpha}=Z_{13}$ fixing only the point $\alpha$, and Line 4 of Table 1 holds.

Finally suppose that one of the LiNEs 32-35 holds. Without loss of generality we may take $T=Z_{5}$ and $S=Z_{53}$. Now $b / \operatorname{gcd}(b, v)=2$ divides $\left|G_{\alpha}\right|$, so $|G|$ is even. By Lemma 2.4(b)(ii), $G_{\alpha}$ contains a unique involution $w$ that inverts $S$ and centralises $T$. Now $w=h^{26}$ where $\langle h\rangle=\operatorname{Aut}(S)$, and we set $\langle g\rangle=\operatorname{Aut}(T)$. Since $b=2 v$, we have $\left|G_{\alpha}\right|=2\left|G_{\lambda}\right|$, and since each involution fixes a line it follows that $\left|G_{\lambda}\right|$ is even and so 4||$G_{\alpha} \mid$. It follows from Lemma 2.4(b) that a Sylow 2-subgroup of $G_{\alpha}$ is cyclic so there is an element $u \in G_{\alpha}$ of order 4 such that $u^{2}=w$. The only possibilities for $u$ are $h^{13}$ and $g^{2} h^{13}$ or their inverses $h^{39}$, $g^{2} h^{39}$ respectively. Thus we may assume that $u=h^{13}$ or $g^{2} h^{13}$. In either case $u$ fixes setwise the set $T=\operatorname{Fix}_{\mathcal{P}}(w) \in \mathfrak{C}^{\prime}$. If $u=h^{13}$ then $u$ fixes $T$ pointwise, while if $u=g^{2} h^{13}$, then $u$ 
fixes $\alpha$ and has two orbits of length 2 in $T$. In either case $u$ fixes setwise a pair $\{\beta, \gamma\}$ of points of $T$. Let $\lambda^{\prime}=\lambda(\beta, \gamma)$ be the line through $\beta$ and $\gamma$. Since the $\mathfrak{C}^{\prime}$-intersection type is $\left(1^{10}, 2\right)$ it follows that $\left|\lambda^{\prime} \cap T\right|=2$, and that $u$ fixes setwise the 10 classes $C^{\prime} \in \mathfrak{C}^{\prime}$ such that $\left|C^{\prime} \cap \lambda^{\prime}\right|=1$. This is a contradiction since $\langle u\rangle$ fixes the class $T \in \mathfrak{C}^{\prime}$ and permutes the remaining 52 classes in orbits of length 4 .

Theorem 1.2 follows from Lemma 4.1 4.4.

\section{Acknowledgments}

The authors are very grateful to Maska Law for running the programs from [3] for line size $k \leq 12$ and some useful discussion. They also thank Anton Betten for conducting the searches on the possibilities in Table 1.

\section{References}

[1] A. Betten and C. E. Praeger, Searching for line-transitive point-imprimitive linear spaces with a grid structure, in preparation.

[2] A. Betten, G. Cresp and C. E. Praeger, Line-transitive point-imprimitive linear spaces: the grid case, submitted.

[3] Anton Betten, Anne Delandtsheer, Maska Law, Alice C. Niemeyer, Cheryl E. Praeger and Shenglin Zhou, Linear spaces with a line-transitive point-imprimitive automorphism group and Fang-Li parameter $\operatorname{gcd}(k, r)$ at most eight, submitted for publication. (available at arxiv:math.CO/0701629)

[4] A. R. Camina and Susanne Mischke, Line-transitive automorphism groups of linear spaces, Electron. J. Combin. 3(1): Research Paper 3, 16 pp. (electronic), 1996.

[5] A. R. Camina and C. E. Praeger, Line-transitive automorphism groups of linear spaces, Bull. London Math. Soc. 25(4),309-315, 1993.

[6] A. R. Camina and J. Siemons, Block transitive automorphism groups of $2-(v, k, 1)$ block designs, J. Combin. Theory Ser.A, 51, 268-276(1989).

[7] A. R. Camina and F. Spiezia, Sporadic groups and Automorphisms of Linear Spaces, J. Combin. Designs, 8, 353-362 (2000).

[8] J. H. Conway, R. T. Curtis, S. P. Norton, R. A. Parker, R. A. Wilson, Atlas of finite groups. Maximal subgroups and ordinary characters for simple groups. With computational assistance from J. G. Thackray. Oxford University Press, Eynsham, 1985.

[9] G. Cresp, Searching for line-transitive, point-imprimitive, linear spaces. Honours Dissertation, The University of Western Australia, Perth, 2001. (available at http://arxiv.org/abs/math.CO/0604532) 
[10] D. H. Davies, Automorphism of Designs, PhD thesis, University of East Anglia, 1987.

[11] A. Delandtsheer and J. Doyen, Most block-transitive $t$-designs are point-primitive. Geom. Dedicata, 29(3):307-310, 1989.

[12] A. Delandtsheer, A. C. Niemeyer, and C. E. Praeger. Finite line-transitive linear spaces: parameters and normal point partitions. Adv. Geom., 3:469-485, 2003.

[13] J. D. Dixon and B. Mortimer, Permutation groups, Springer-Verlag, New York, 1996.

[14] The GAP Group, GAP - Groups, Algorithms, and Programming, Version 4.4; 2004, (\protect\vrule width0pt \protect \href\{http://www.gap-system.org\}\{http://www.gap-syst

[15] C. W. H. Lam, L. Thiel, and S. Swiercz, The nonexistence of finite projective planes of order 10, Canad. J. Math. 41 (1989), no. 6, 1117-1123.

[16] W. Nickel, A. C. Niemeyer, C. M. O'Keefe, T. Penttila, and C. E. Praeger, The blocktransitive, point-imprimitive 2-(729, 8, 1) designs, Appl. Algebra Engrg. Comm. Comput. 3 (1992), 47-61.

[17] C. M. O'Keefe, T. Penttila and C. E. Praeger, Block-transitive, point-imprimitive designs with $\lambda=1$, Discrete Mathematics 115(1993), 231-244.

[18] C. E. Praeger and Ngo Dac Tuan, Inequalities involving the Delandtsheer-Doyen parameters for finite line-transitive linear spaces, J. Combin. Theory Ser.A, 102(1):268276(2003).

[19] S. D. Stoichev and V. D. Tonchev, The automorphism groups of the known 2-(91, 6, 1) designs. C. R. Acad. Bulgare Sci. 41 (1988), no. 4, 15-16. 


\begin{tabular}{|clccccc|}
\hline LINE & $v=d \cdot c$ & $x$ & $y$ & $k$ & $b / v$ & INTER-TYPE \\
\hline \hline 1 & $145=5 \cdot 29$ & 7 & 1 & 9 & 2 & $\left(1,2,3^{2}\right)$ \\
2 & $145=5 \cdot 29$ & 7 & 1 & 9 & 2 & $\left(1^{3}, 2,4\right)$ \\
3 & $145=29 \cdot 5$ & 1 & 7 & 9 & 2 & $\left(1^{7}, 2\right)$ \\
4 & $217=7 \cdot 31$ & 5 & 1 & 9 & 3 & $\left(1^{2}, 2^{2}, 3\right)$ \\
5 & $217=31 \cdot 7$ & 1 & 5 & 9 & 3 & $\left(1^{7}, 2\right)$ \\
6 & $289=17 \cdot 17$ & 2 & 2 & 9 & 4 & $\left(1^{5}, 2^{2}\right)$ \\
\hline \hline 7 & $91=7 \cdot 13$ & 6 & 3 & 10 & 1 & $\left(1,2^{3}, 3\right)$ \\
8 & $91=7 \cdot 13$ & 6 & 3 & 10 & 1 & $\left(1^{4}, 3^{2}\right)$ \\
9 & $91=7 \cdot 13$ & 6 & 3 & 10 & 1 & $\left(1^{6}, 4\right)$ \\
10 & $91=13 \cdot 7$ & 3 & 6 & 10 & 1 & $\left(1^{4}, 2^{3}\right)$ \\
11 & $91=13 \cdot 7$ & 3 & 6 & 10 & 1 & $\left(1^{7}, 3\right)$ \\
12 & $451=11 \cdot 41$ & 4 & 1 & 10 & 5 & $\left(1^{2}, 2^{4}\right)$ \\
13 & $451=11 \cdot 41$ & 4 & 1 & 10 & 5 & $\left(1^{5}, 2,3\right)$ \\
14 & $451=41 \cdot 11$ & 1 & 4 & 10 & 5 & $\left(1^{8}, 2\right)$ \\
\hline \hline 15 & $111=37 \cdot 3$ & 1 & 18 & 11 & 1 & $\left(1^{9}, 2\right)$ \\
16 & $221=13 \cdot 17$ & 4 & 3 & 11 & 2 & $\left(1^{3}, 2^{4}\right)$ \\
17 & $221=13 \cdot 17$ & 4 & 3 & 11 & 2 & $\left(1^{6}, 2,3\right)$ \\
18 & $221=17 \cdot 13$ & 3 & 4 & 11 & 2 & $\left(1^{5}, 2^{3}\right)$ \\
19 & $221=17 \cdot 13$ & 3 & 4 & 11 & 2 & $\left(1^{8}, 3\right)$ \\
20 & $441=9 \cdot 49$ & 6 & 1 & 11 & 4 & $\left(1^{2}, 2^{3}, 3\right)$ \\
21 & $441=9 \cdot 49$ & 6 & 1 & 11 & 4 & $\left(1^{5}, 3^{2}\right)$ \\
22 & $441=9 \cdot 49$ & 6 & 1 & 11 & 4 & $\left(1^{7}, 4\right)$ \\
23 & $441=49 \cdot 9$ & 1 & 6 & 11 & 4 & $\left(1^{9}, 2\right)$ \\
24 & $1431=27 \cdot 53$ & 2 & 1 & 11 & 13 & $\left(1^{7}, 2^{2}\right)$ \\
25 & $1431=53 \cdot 27$ & 1 & 2 & 11 & 13 & $\left(1^{9}, 2\right)$ \\
\hline \hline 26 & $133=7 \cdot 19$ & 9 & 3 & 12 & 1 & $\left(2^{3}, 3^{2}\right)$ \\
27 & $133=7 \cdot 19$ & 9 & 3 & 12 & 1 & $\left(1^{3}, 3^{3}\right)$ \\
28 & $133=7 \cdot 19$ & 9 & 3 & 12 & 1 & $\left(1^{2}, 2^{3}, 4\right)$ \\
29 & $133=7 \cdot 19$ & 9 & 3 & 12 & 1 & $\left(1^{5}, 3,4\right)$ \\
30 & $133=19 \cdot 7$ & 3 & 9 & 12 & 1 & $\left(1^{6}, 2^{3}\right)$ \\
31 & $133=19 \cdot 7$ & 3 & 9 & 12 & 1 & $\left(1^{9}, 3\right)$ \\
32 & $265=5 \cdot 53$ & 13 & 1 & 12 & 2 & $\left(2,3^{2}, 4\right)$ \\
33 & $265=5 \cdot 53$ & 13 & 1 & 12 & 2 & $\left(1^{2}, 2,4^{2}\right)$ \\
34 & $265=5 \cdot 53$ & 13 & 1 & 12 & 2 & $\left(1,2^{3}, 5\right)$ \\
36 & $265=53 \cdot 5$ & 1 & 13 & 12 & 2 & $\left(1^{10}, 2\right)$ \\
37 & $793=13 \cdot 61$ & 5 & 1 & 12 & 6 & $\left(1^{2}, 2^{5}\right)$ \\
38 & $793=61 \cdot 13$ & 1 & 5 & 12 & 6 & $\left(1^{10}, 2\right)$ \\
\hline
\end{tabular}

Table 3: Parameter Sets for $9 \leq k \leq 12$ 


\begin{tabular}{|c|c|c|}
\hline LINE & Group $G^{\mathcal{C}}$ & Group $G^{C}$ \\
\hline $\begin{array}{l}1-2 \\
3 \\
4 \\
5 \\
6 \\
\end{array}$ & $\begin{array}{l}5:[2 a], \text { where } a \mid 2, \text { or } A_{5}, S_{5} \\
29:[2 a], \text { where } a \mid 14 \\
7:[3 a], \text { where } a \mid 2, \text { or } L_{3}(2) \\
31:[3 a], \text { where } a=1,2,5 \\
17:[4 a], \text { where } a \mid 2 \\
\end{array}$ & $\begin{array}{l}29:[2 b], \text { where } b=1,2,7 \\
5:[2 b], \text { where } b \mid 2 \\
31:[3 b], \text { where } b=1,2,5 \\
7:[3 b], \text { where } b \mid 2 \\
17:[4 b], \text { where } b \mid 4 \\
\end{array}$ \\
\hline $\begin{array}{l}7 \\
8 \\
9 \\
10 \\
11 \\
12 \\
13 \\
14\end{array}$ & $\begin{array}{l}7:[a], a=1,2,3 \\
7:[a], a=1,2,3 \\
7:[a], a \mid 6 \text { or } L_{3}(2), A_{7}, S_{7} \\
13:[a], a=1,2,3,4,6 \\
13:[a], a=1,2,3,4,6 \\
11:[5 a], \text { where } a \mid 2, \text { or } L_{2}(11) \\
11:[5 a], \text { where } a \mid 2, \text { or } L_{2}(11) \\
41:[5 a], \text { where } a \mid 8\end{array}$ & $\begin{array}{l}13:[b], \text { where } b=1,2,3,4,6 \\
13:[b], \text { where } b \mid 12 \text { or } L_{3}(3) \\
13:[b], \text { where } b \mid 12 \text { or } L_{3}(3) \\
7:[b], \text { where } a \mid 6 \\
7:[b], \text { where } b \mid 6 \text { or } L_{3}(2) \\
41:[5 b], \text { where } b \mid 8 \\
41:[5 b], \text { where } b \mid 4 \\
11:[5 b], \text { where } b \mid 2\end{array}$ \\
\hline $\begin{array}{l}15 \\
16 \\
17 \\
18 \\
19 \\
20 \\
21-22 \\
23 \\
24 \\
25 \\
\end{array}$ & $\begin{array}{l}37:[a], \text { where } a=4,12 \text { or } a \mid 18 \\
13:[2 a], \text { where } a \mid 6, \text { or } L_{3}(3) \\
13:[2 a], \text { where } a=1,2,3 \\
17:[2 a], \text { where } a \mid 4 \\
17:[2 a], \text { where } a \mid 8 \\
\text { affine or } \operatorname{Soc}\left(G^{\mathfrak{c}}\right)=L_{2}(8) \text { or } A_{9} \\
\text { affine or } \operatorname{Soc}\left(G^{\mathfrak{c}}\right)=L_{2}(8) \text { or } A_{9} \\
\text { affine or } \operatorname{Soc}\left(G^{\mathfrak{c}}\right)=L_{3}(2)^{2} \text { or } A_{7}^{2} \\
\text { affine } \\
53:[13 a], \text { where } a \mid 2\end{array}$ & $\begin{array}{l}3:[b], \text { where } b \mid 2 \\
17:[2 b], \text { where } b \mid 8 \\
17:[2 b], \text { where } b \mid 4 \\
13:[2 b], \text { where } b \mid 6 \\
13:[2 b], \text { where } b \mid 6 \\
\text { affine } \\
\text { affine } \\
\text { affine } \\
53:[13 b], \text { where } b \mid 4 \\
\text { affine }\end{array}$ \\
\hline $\begin{array}{l}26,28,29 \\
27 \\
30 \\
31 \\
32-34 \\
35 \\
36 \\
37 \\
38\end{array}$ & $\begin{array}{l}7:[a], \text { where } a=1,2,3 \\
7:[a], \text { where } a \mid 6 \text { or } L_{3}(2) \\
19:[a], \text { where } a \mid 18 \text { and } a<18 \\
19:[a], \text { where } a \mid 18 \text { and } a<18 \\
5:[2 a], \text { where } a \mid 2, \text { or } A_{5}, S_{5} \\
53:[2 a], \text { where } a=1,2,13 \\
13:[6 a], \text { where } a \mid 2, \text { or } L_{3}(3) \\
13:[6 a], \text { where } a \mid 2, \text { or } L_{3}(3) \\
61:[6 a], \text { where } a \mid 10\end{array}$ & $\begin{array}{l}19:[b], \text { where } b \mid 18 \text { and } b<18 \\
19:[b], \text { where } b \mid 18 \\
7:[b], \text { where } b \mid 6 \\
7:[b], \text { where } b \mid 6 \\
53:[2 b], \text { where } b=1,2,13 \\
5:[2 b], \text { where } b \mid 2 \\
61:[6 b], \text { where } b \mid 10 \\
61:[6 b], \text { where } b=1,2,5 \\
13:[6 b], \text { where } b \mid 2 \\
\end{array}$ \\
\hline
\end{tabular}

Table 4: Group information for $9 \leq k \leq 12$ 